Supplement of Proc. IAHS, 379, 415-420, 2018

https://doi.org/10.5194/piahs-379-415-2018-supplement

(c) Author(s) 2018. This work is distributed under

the Creative Commons Attribution 4.0 License.

(c) (1)

Supplement of

\title{
Socio-hydrological implications of water management in the dry zone of Sri Lanka
}

Isurun Upeksha Gamage and Hetti Arachchige Hemachandra Jayasena

Correspondence to: Isurun Upeksha Gamage (isurun.gamage@yahoo.com)

The copyright of individual parts of the supplement might differ from the CC BY 4.0 License. 


\section{GN Division Area}

Weearakodiyana

Weearakodiyana

Weearakodiyana

Weearakodiyana

Weearakodiyana

Weearakodiyana

Weearakodiyana

Weearakodiyana

Weearakodiyana

Weearakodiyana

Weearakodiyana

Weearakodiyana

Weearakodiyana

Weearakodiyana

Weearakodiyana

Weearakodiyana

Weearakodiyana

Weearakodiyana

Weearakodiyana

Weearakodiyana

Weearakodiyana

Weearakodiyana

Weearakodiyana

Weearakodiyana

Weearakodiyana

Weearakodiyana

Weearakodiyana

Weearakodiyana

Weearakodiyana

Weearakodiyana

Weearakodiyana

Weearakodiyana

Weearakodiyana

Weearakodiyana

Weearakodiyana

Weearakodiyana

Weearakodiyana

Weearakodiyana

Weearakodiyana

Weearakodiyana

Weearakodiyana

Weearakodiyana

Weearakodiyana

Weearakodiyana

Weearakodiyana

\section{Nationality}

Sinhala

Sinhala

Sinhala

Sinhala

Sinhala

Sinhala

Sinhala

Sinhala

Sinhala

Sinhala

Sinhala

Sinhala

Sinhala

Sinhala

Sinhala

Sinhala

Sinhala

Sinhala

Sinhala

Sinhala

Sinhala

Sinhala

Sinhala

Sinhala

Sinhala

Sinhala

Sinhala

Sinhala

Sinhala

Sinhala

Sinhala

Sinhala

Sinhala

Sinhala

Sinhala

Sinhala

Sinhala

Sinhala

Sinhala

Sinhala

Sinhala

Sinhala

Sinhala

Sinhala

Sinhala lumber of family membes Number of males

3

1

2

2

0

2

1

2

1

2

1

3

2

1

1

2

1

3

2

2

1

1

1

2

3

0

0

0

2

3

0

3

1

2

2

2

1

1

1

2

2

2

1

2

2 


\begin{tabular}{|c|c|c|}
\hline Rekogama & Sinhala & 5 \\
\hline Rekogama & Sinhala & 7 \\
\hline Rekogama & Sinhala & 4 \\
\hline Rekogama & Sinhala & 5 \\
\hline Rekogama & Sinhala & 5 \\
\hline Rekogama & Sinhala & 5 \\
\hline Rekogama & Sinhala & 3 \\
\hline Rekogama & Sinhala & 2 \\
\hline Rekogama & Sinhala & 4 \\
\hline Rekogama & Sinhala & 5 \\
\hline Rekogama & Sinhala & 2 \\
\hline Rekogama & Sinhala & 4 \\
\hline Rekogama & Sinhala & 4 \\
\hline Rekogama & Sinhala & 3 \\
\hline Rekogama & Sinhala & 4 \\
\hline Rekogama & Sinhala & 5 \\
\hline Rekogama & Sinhala & 5 \\
\hline Rekogama & Sinhala & 4 \\
\hline Rekogama & Sinhala & 4 \\
\hline Rekogama & Sinhala & 4 \\
\hline Rekogama & Sinhala & 6 \\
\hline Rekogama & Sinhala & 5 \\
\hline Rekogama & Sinhala & 6 \\
\hline Rekogama & Sinhala & 7 \\
\hline Rekogama & Sinhala & 4 \\
\hline Rekogama & Sinhala & 5 \\
\hline Rekogama & Sinhala & 3 \\
\hline Rekogama & Sinhala & 3 \\
\hline Rekogama & Sinhala & 2 \\
\hline Rekogama & Sinhala & 5 \\
\hline Rekogama & Sinhala & 6 \\
\hline Rekogama & Sinhala & 2 \\
\hline
\end{tabular}




\begin{tabular}{|c|c|c|}
\hline Rekogama & Sinhala & 5 \\
\hline Rekogama & Sinhala & 4 \\
\hline Rekogama & Sinhala & 3 \\
\hline Rekogama & Sinhala & 4 \\
\hline Rekogama & Sinhala & 3 \\
\hline Rekogama & Sinhala & 3 \\
\hline Rekogama & Sinhala & 3 \\
\hline Rekogama & Sinhala & 4 \\
\hline Rekogama & Sinhala & 4 \\
\hline Rekogama & Sinhala & 5 \\
\hline Rekogama & Sinhala & 13 \\
\hline Rekogama & Sinhala & 5 \\
\hline Rekogama & Sinhala & 3 \\
\hline Rekogama & Sinhala & 2 \\
\hline Rekogama & Sinhala & 4 \\
\hline Rekogama & Sinhala & 4 \\
\hline Rekogama & Sinhala & 8 \\
\hline Rekogama & Sinhala & 2 \\
\hline Aladeniya & Sinhala & 5 \\
\hline Aladeniya & Sinhala & 3 \\
\hline Aladeniya & Sinhala & 3 \\
\hline Aladeniya & Sinhala & 5 \\
\hline Aladeniya & Sinhala & 5 \\
\hline Aladeniya & Sinhala & 4 \\
\hline Aladeniya & Sinhala & 4 \\
\hline Aladeniya & Sinhala & 4 \\
\hline Aladeniya & Sinhala & 4 \\
\hline Aladeniya & Sinhala & 4 \\
\hline Aladeniya & Sinhala & 3 \\
\hline Aladeniya & Sinhala & 3 \\
\hline Aladeniya & Sinhala & 6 \\
\hline Aladeniya & Sinhala & 5 \\
\hline
\end{tabular}




\begin{tabular}{|c|c|c|}
\hline Aladeniya & Sinhala & 4 \\
\hline Aladeniya & Sinhala & 3 \\
\hline Aladeniya & Sinhala & 7 \\
\hline Aladeniya & Sinhala & 3 \\
\hline Aladeniya & Sinhala & 2 \\
\hline Aladeniya & Sinhala & 7 \\
\hline Aladeniya & Sinhala & 4 \\
\hline Aladeniya & Sinhala & 3 \\
\hline Aladeniya & Sinhala & 3 \\
\hline Aladeniya & Sinhala & 5 \\
\hline Aladeniya & Sinhala & 7 \\
\hline Aladeniya & Sinhala & 7 \\
\hline Aladeniya & Sinhala & 2 \\
\hline Aladeniya & Sinhala & 5 \\
\hline Aladeniya & Sinhala & 4 \\
\hline Hedeniya & Sinhala & 4 \\
\hline Hedeniya & Sinhala & 5 \\
\hline Hedeniya & Sinhala & 9 \\
\hline Hedeniya & Sinhala & 4 \\
\hline Hedeniya & Sinhala & 4 \\
\hline Hedeniya & Sinhala & 8 \\
\hline Hedeniya & Sinhala & 2 \\
\hline Hedeniya & Sinhala & 5 \\
\hline Hedeniya & Sinhala & 4 \\
\hline Hedeniya & Sinhala & 5 \\
\hline Hedeniya & Sinhala & 7 \\
\hline Hedeniya & Sinhala & 2 \\
\hline Hedeniya & Sinhala & 4 \\
\hline Hedeniya & Muslim & 2 \\
\hline Hedeniya & Tamil & 6 \\
\hline Hedeniya & Muslim & 6 \\
\hline Hedeniya & Sinhala & 3 \\
\hline
\end{tabular}




\begin{tabular}{|c|c|c|}
\hline Hedeniya & Sinhala & 2 \\
\hline Hedeniya & Sinhala & 4 \\
\hline Hedeniya & Sinhala & 5 \\
\hline Hedeniya & Sinhala & 2 \\
\hline Hedeniya & Sinhala & 3 \\
\hline Hedeniya & Sinhala & 5 \\
\hline Hedeniya & Sinhala & 6 \\
\hline Hedeniya & Sinhala & 6 \\
\hline Hedeniya & Sinhala & 6 \\
\hline Hedeniya & Sinhala & 2 \\
\hline Hedeniya & Sinhala & 5 \\
\hline Hedeniya & Sinhala & 5 \\
\hline Hedeniya & Sinhala & 6 \\
\hline Hedeniya & Sinhala & 5 \\
\hline Hedeniya & Sinhala & 2 \\
\hline Hedeniya & Sinhala & 5 \\
\hline Hedeniya & Sinhala & 4 \\
\hline Hedeniya & Sinhala & 3 \\
\hline Hedeniya & Sinhala & 6 \\
\hline Hedeniya & Sinhala & 5 \\
\hline Hedeniya & Sinhala & 7 \\
\hline Hedeniya & Sinhala & 4 \\
\hline Hedeniya & Sinhala & 6 \\
\hline Hedeniya & Sinhala & 5 \\
\hline Hedeniya & Sinhala & 4 \\
\hline Hedeniya & Sinhala & 7 \\
\hline Hedeniya & Sinhala & 4 \\
\hline Hedeniya & Sinhala & 4 \\
\hline Hedeniya & Sinhala & 4 \\
\hline Hedeniya & Sinhala & 5 \\
\hline Hedeniya & Sinhala & 5 \\
\hline Chillaw & Sinhala & 1 \\
\hline Chillaw & Burger & 6 \\
\hline Chillaw & Sinhala & 2 \\
\hline Chillaw & Sinhala & 2 \\
\hline Chillaw & Sinhala & 3 \\
\hline Chillaw & Sinhala & 5 \\
\hline
\end{tabular}




\begin{tabular}{|c|c|c|}
\hline Chillaw & Tamil & 4 \\
\hline Chillaw & Sinhala & 2 \\
\hline Chillaw & Tamil & 4 \\
\hline Chillaw & Tamil & 2 \\
\hline Chillaw & Tamil & 2 \\
\hline Chillaw & Sinhala & 4 \\
\hline Chillaw & Sinhala & 3 \\
\hline Chillaw & Sinhala & 3 \\
\hline Chillaw & Sinhala & 1 \\
\hline Chillaw & Sinhala & 5 \\
\hline Chillaw & Tamil & 4 \\
\hline Chillaw & Sinhala & 6 \\
\hline Chillaw & Sinhala & 4 \\
\hline Chillaw & Sinhala & 3 \\
\hline Chillaw & Sinhala & 3 \\
\hline Chillaw & Sinhala & 5 \\
\hline Chillaw & Sinhala & 6 \\
\hline Chillaw & Sinhala & 4 \\
\hline Chillaw & Burger & 4 \\
\hline Chillaw & Sinhala & 1 \\
\hline Chillaw & Sinhala & 1 \\
\hline Chillaw & Sinhala & 4 \\
\hline Chillaw & Sinhala & 5 \\
\hline Chillaw & Sinhala & 4 \\
\hline Chillaw & Burger & 3 \\
\hline Chillaw & Sinhala & 2 \\
\hline Chillaw & Sinhala & 3 \\
\hline Chillaw & Sinhala & 4 \\
\hline Chillaw & Sinhala & 6 \\
\hline Chillaw & Sinhala & 5 \\
\hline Chillaw & Sinhala & 2 \\
\hline Chillaw & Sinhala & 3 \\
\hline Chillaw & Sinhala & 5 \\
\hline Chillaw & Sinhala & 5 \\
\hline Chillaw & Sinhala & 5 \\
\hline Chillaw & Sinhala & 4 \\
\hline Chillaw & Sinhala & 3 \\
\hline
\end{tabular}




$\begin{array}{lccc}\text { Chillaw } & \text { Sinhala } & 4 & 1 \\ \text { Chillaw } & \text { Sinhala } & 6 & 3 \\ \text { Chillaw } & \text { Sinhala } & 9 & 4 \\ \text { Chillaw } & \text { Sinhala } & 6 & 3 \\ \text { Chillaw } & \text { Sinhala } & 5 & 3 \\ \text { Chillaw } & \text { Sinhala } & 4 & 2 \\ \text { Chillaw } & \text { Sinhala } & 4 & 1 \\ & & & \\ & & 13 & \end{array}$


Number of females Highest education level in the family 2 2
Higher Education

Grade 6 to O/L

$$
\text { Up to } A / L
$$

Higher Education

Grade 6 to O/L Up to $A / L$

Grade 6 to O/L

Up to Grade Five

Up to $A / L$

Up to $A / L$

Grade 6 to O/L

Grade 6 to O/L

Grade 6 to $\mathrm{O} / \mathrm{L}$

Grade 6 to O/L

Up to Grade Five

Up to $A / L$

Up to $A / L$

Grade 6 to $\mathrm{O} / \mathrm{L}$

Grade 6 to O/L

Higher Education Up to A/L

Up to Grade Five

Up to Grade Five

Grade 6 to O/L

Grade 6 to O/L

Grade 6 to O/L

Grade 6 to O/L

Up to Grade Five

Up to $A / L$

Up to $A / L$

Grade 6 to $\mathrm{O} / \mathrm{L}$

Grade 6 to O/L

Grade 6 to $\mathrm{O} / \mathrm{L}$

$$
\text { Up to } A / L
$$

Up to $A / L$

Up to $A / L$

Up to $A / L$

Grade 6 to $\mathrm{O} / \mathrm{L}$

Up to $A / L$

Up to $A / L$

Grade 6 to O/L

Higher Education

Higher Education

$$
\text { Up to } A / L
$$

Higher Education
Total Years of Eucation 
Grade 6 to O/L

Higher Education $\quad 16$

Higher Education $\quad 16$

Up to A/L 12

Grade 6 to O/L 9

Up to Grade Five 3

Formal Schooling 13

Grade 6 to O/L 9

Grade 6 to O/L 9

Grade 6 to $\mathrm{O} / \mathrm{L}$

Grade 6 to $\mathrm{O} / \mathrm{L}$

Up to Grade Five 3

Up to $A / L \quad 12$

Grade 6 to $\mathrm{O} / \mathrm{L}$

Up to $A / L$

Up to $A / L \quad 12$

Up to Grade Five 3

Grade 6 to O/L 9

Grade 6 to O/L 9

Grade 6 to O/L 9 
12

12

12

Grade 6 to O/L 9

Up to A/L 12

Grade 6 to O/L 9

Up to $A / L$

Up to Grade Five 3

Grade 6 to O/L 9

Up to $A / L$

Up to $A / L \quad 12$

Up to $A / L \quad 12$

Higher Education $\quad 16$

Grade 6 to O/L 9

Up to A/L 12

Grade 6 to O/L 9

Up to $A / L$

Higher Education $\quad 16$

Higher Education $\quad 16$

Up to $A / L$

Grade 6 to O/L 9

Grade 6 to O/L 9

Up to $A / L \quad 12$ 
12

12

12

Grade 6 to $\mathrm{O} / \mathrm{L}$

Grade 6 to O/L 9

Higher Education $\quad 16$

Grade 6 to O/L 9

Up to A/L 12

Grade 6 to $\mathrm{O} / \mathrm{L}$

Up to $A / L \quad 12$

Up to $A / L$

Grade 6 to O/L 9

Higher Education $\quad 16$

Grade 6 to O/L 9

Grade 6 to O/L 9

Grade 6 to O/L 9

Grade 6 to O/L 9

Up to $A / L$

Up to A/L 12

Higher Education $\quad 16$

Up to $A / L$

Up to $A / L \quad 12$

Grade 6 to O/L 9

Grade 6 to O/L 9

Grade 6 to O/L 9 
Grade 6 to O/L 9

Up to Grade Five 3

Grade 6 to $\mathrm{O} / \mathrm{L}$

Grade 6 to $\mathrm{O} / \mathrm{L}$

Up to Grade Five 3

Up to Grade Five 3

Up to Grade Five 3

Up to Grade Five 3

Grade 6 to O/L 9

Grade 6 to $\mathrm{O} / \mathrm{L}$

Grade 6 to $\mathrm{O} / \mathrm{L}$

Up to Grade Five 3

Up to Grade Five 3

Up to Grade Five 3

Up to Grade Five 3

Grade 6 to $\mathrm{O} / \mathrm{L}$

Grade 6 to $\mathrm{O} / \mathrm{L}$

Higher Education 16

Grade 6 to O/L 9

Up to $A / L \quad 12$

Just Studying 9

Grade 6 to $\mathrm{O} / \mathrm{L}$

Grade 6 to $\mathrm{O} / \mathrm{L}$

Grade 6 to $\mathrm{O} / \mathrm{L}$

Up to Grade Five 3

Grade 6 to $\mathrm{O} / \mathrm{L}$

Up to Grade Five 3

Grade 6 to $\mathrm{O} / \mathrm{L}$

Grade 6 to $\mathrm{O} / \mathrm{L}$

Up to $A / L \quad 12$

Up to $A / L$

Higher Education 16

Up to $A / L \quad 12$

Grade 6 to $\mathrm{O} / \mathrm{L}$ 
Grade 6 to O/L 9

Up to $A / L \quad 12$

Grade 6 to O/L 9

Up to Grade Five 3

Grade 6 to $\mathrm{O} / \mathrm{L}$

Grade 6 to $\mathrm{O} / \mathrm{L}$

Grade 6 to $\mathrm{O} / \mathrm{L}$

Grade 6 to O/L 9

Grade 6 to O/L 9

Grade 6 to O/L 9

Grade 6 to $\mathrm{O} / \mathrm{L}$

Grade 6 to $\mathrm{O} / \mathrm{L}$

Grade 6 to O/L 9

Up to $A / L$

Up to $A / L \quad 12$

Grade 6 to $O / L$

Up to $A / L$

Grade 6 to $\mathrm{O} / \mathrm{L}$

Grade 6 to $\mathrm{O} / \mathrm{L}$

Grade 6 to $\mathrm{O} / \mathrm{L}$

Grade 6 to $\mathrm{O} / \mathrm{L}$

Up to $A / L \quad 12$

Up to $A / L \quad 12$

Up to A/L 12

Up to $A / L \quad 12$

Grade 6 to $\mathrm{O} / \mathrm{L}$

Up to $A / L \quad 12$

Grade 6 to $\mathrm{O} / \mathrm{L}$

Up to $A / L \quad 12$

Up to $A / L \quad 12$

Up to $A / L \quad 12$

Up to $A / L$

Up to $A / L \quad 12$

Up to $A / L \quad 12$

Up to $A / L \quad 12$

Grade 6 to $\mathrm{O} / \mathrm{L}$

Grade 6 to $\mathrm{O} / \mathrm{L}$ 


\begin{tabular}{|c|c|c|c|}
\hline paterfamilias job & \multicolumn{2}{|c|}{ paterfamilias additional incoment Aid (Samurdi/Ja } & Monthly Income \\
\hline Government & none & no & $15000-20000$ \\
\hline Business & none & yes & $5000-10000$ \\
\hline Government & none & no & $10000-15000$ \\
\hline Government & none & no & OVER 20000 \\
\hline Self Employment & none & yes & below 5000 \\
\hline Government & none & no & $10000-15000$ \\
\hline Government & none & no & $5000-10000$ \\
\hline Private Sector & none & yes & below 5000 \\
\hline Private Sector & none & no & $5000-10000$ \\
\hline Industrial Sector & none & no & below 5000 \\
\hline Agriculture & none & yes & below 5000 \\
\hline Industrial Sector & none & yes & below 5000 \\
\hline Private Sector & none & no & below 5000 \\
\hline Industrial Sector & none & yes & below 5000 \\
\hline Industrial Sector & none & yes & below 5000 \\
\hline Industrial Sector & none & yes & $5000-10000$ \\
\hline Private Sector & none & yes & below 5000 \\
\hline Industrial Sector & none & no & below 5000 \\
\hline Private Sector & none & yes & below 5000 \\
\hline Agriculture & none & no & $10000-15000$ \\
\hline Government & none & no & $5000-10000$ \\
\hline Industrial Sector & none & yes & below 5000 \\
\hline Industrial Sector & none & yes & below 5000 \\
\hline Industrial Sector & none & no & $10000-15000$ \\
\hline Business & none & no & $10000-15000$ \\
\hline Government & none & no & $10000-15000$ \\
\hline Private Sector & none & no & below 5000 \\
\hline Industrial Sector & none & yes & below 5000 \\
\hline Industrial Sector & none & no & $5000-10000$ \\
\hline Government & none & yes & $5000-10000$ \\
\hline Industrial Sector & none & yes & below 5000 \\
\hline Industrial Sector & none & no & below 5000 \\
\hline Private Sector & none & yes & below 5000 \\
\hline Agriculture & none & yes & below 5000 \\
\hline Private Sector & none & no & $5000-10000$ \\
\hline Government & none & no & $15000-20000$ \\
\hline Government & none & no & $5000-10000$ \\
\hline Government & none & yes & below 5000 \\
\hline Industrial Sector & none & yes & below 5000 \\
\hline Government & none & no & $10000-15000$ \\
\hline Private Sector & none & yes & below 5000 \\
\hline Government & none & no & $5000-10000$ \\
\hline Private Sector & none & yes & below 5000 \\
\hline Agriculture & none & yes & below 5000 \\
\hline Agriculture & none & yes & $10000-15000$ \\
\hline
\end{tabular}




\begin{tabular}{|c|c|c|c|}
\hline Industrial Sector & Agriculture & no & $5000-10000$ \\
\hline Agriculture & none & no & $10000-15000$ \\
\hline Government & Agriculture & no & $5000-10000$ \\
\hline Government & Agriculture & no & $5000-10000$ \\
\hline Government & Agriculture & no & $5000-10000$ \\
\hline Government & none & no & $5000-10000$ \\
\hline Private Sector & Agriculture & no & $10000-15000$ \\
\hline Government & Agriculture & no & $15000-20000$ \\
\hline Government & Agriculture & no & $10000-15000$ \\
\hline Government & Agriculture & no & $5000-10000$ \\
\hline Government & Agriculture & yes & below 5000 \\
\hline Government & none & no & $5000-10000$ \\
\hline Agriculture & none & no & $5000-10000$ \\
\hline Government & Agriculture & no & $5000-10000$ \\
\hline Self Employment & Agriculture & no & $5000-10000$ \\
\hline Industrial Sector & Agriculture & no & $5000-10000$ \\
\hline Agriculture & none & yes & below 5000 \\
\hline Agriculture & Agriculture & no & $5000-10000$ \\
\hline Government & none & no & $10000-15000$ \\
\hline Agriculture & none & no & $5000-10000$ \\
\hline Agriculture & none & no & $5000-10000$ \\
\hline Industrial Sector & Agriculture & no & $5000-10000$ \\
\hline Industrial Sector & Agriculture & no & $5000-10000$ \\
\hline Industrial Sector & Agriculture & no & $5000-10000$ \\
\hline Government & Agriculture & no & $5000-10000$ \\
\hline Industrial Sector & none & yes & below 5000 \\
\hline Government & Agriculture & no & $10000-15000$ \\
\hline Government & Agriculture & yes & below 5000 \\
\hline Agriculture & none & yes & below 5000 \\
\hline Agriculture & none & yes & below 5000 \\
\hline Agriculture & none & yes & below 5000 \\
\hline Foreign & Agriculture & no & $10000-15000$ \\
\hline
\end{tabular}




\begin{tabular}{|c|c|c|c|}
\hline Agriculture & none & yes & below 5000 \\
\hline Government & Agriculture & no & $5000-10000$ \\
\hline Agriculture & none & yes & below 5000 \\
\hline Agriculture & Business & yes & below 5000 \\
\hline Foreign & none & yes & below 5000 \\
\hline Industrial Sector & none & yes & below 5000 \\
\hline Private Sector & none & yes & below 5000 \\
\hline Government & none & yes & below 5000 \\
\hline Agriculture & none & yes & below 5000 \\
\hline Government & none & no & $5000-10000$ \\
\hline Business & Agriculture & no & $5000-10000$ \\
\hline Government & none & no & $5000-10000$ \\
\hline Industrial Sector & Agriculture & yes & below 5000 \\
\hline Agriculture & none & no & $5000-10000$ \\
\hline Agriculture & none & yes & below 5000 \\
\hline Government & Agriculture & no & $10000-15000$ \\
\hline Agriculture & none & yes & below 5000 \\
\hline Agriculture & none & yes & below 5000 \\
\hline Government & none & no & below 5000 \\
\hline Government & none & no & OVER 20000 \\
\hline Government & none & no & $10000-15000$ \\
\hline Government & none & no & $15000-20000$ \\
\hline Business & none & no & $5000-10000$ \\
\hline Government & none & no & $15000-20000$ \\
\hline Private Sector & none & no & $5000-10000$ \\
\hline Private Sector & none & no & $10000-15000$ \\
\hline Government & none & no & OVER 20000 \\
\hline Government & none & no & OVER 20000 \\
\hline Private Sector & none & no & $5000-10000$ \\
\hline Private Sector & none & no & $10000-15000$ \\
\hline Business & none & no & $10000-15000$ \\
\hline Private Sector & none & no & $10000-15000$ \\
\hline
\end{tabular}




\begin{tabular}{|c|c|c|c|}
\hline Government & none & no & OVER 20000 \\
\hline Government & none & no & $10000-15000$ \\
\hline Government & none & no & OVER 20000 \\
\hline Government & none & no & $15000-20000$ \\
\hline Government & none & no & $15000-20000$ \\
\hline Private Sector & none & no & $10000-15000$ \\
\hline Government & none & no & OVER 20000 \\
\hline Government & none & no & $10000-15000$ \\
\hline Agriculture & none & no & $10000-15000$ \\
\hline Business & none & no & $10000-15000$ \\
\hline Business & none & no & $10000-15000$ \\
\hline Business & none & no & OVER 20000 \\
\hline Government & none & no & OVER 20000 \\
\hline Business & none & no & $10000-15000$ \\
\hline Business & none & no & OVER 20000 \\
\hline Government & none & no & $5000-10000$ \\
\hline Agriculture & none & no & $5000-10000$ \\
\hline Private Sector & none & no & $10000-15000$ \\
\hline Private Sector & none & no & $5000-10000$ \\
\hline Government & none & no & $5000-10000$ \\
\hline Industrial Sector & none & no & $15000-20000$ \\
\hline Business & none & no & below 5000 \\
\hline Agriculture & none & no & below 5000 \\
\hline Private Sector & none & no & below 5000 \\
\hline Agriculture & none & no & $5000-10000$ \\
\hline Government & none & no & $15000-20000$ \\
\hline Government & none & no & $5000-10000$ \\
\hline Private Sector & none & no & $5000-10000$ \\
\hline Government & none & no & $5000-10000$ \\
\hline Government & none & no & below 5000 \\
\hline Government & none & no & $5000-10000$ \\
\hline Industrial Sector & none & no & below 5000 \\
\hline
\end{tabular}




\begin{tabular}{|c|c|c|c|}
\hline Government & none & no & $5000-10000$ \\
\hline Industrial Sector & none & no & below 5000 \\
\hline Industrial Sector & none & no & below 5000 \\
\hline Private Sector & none & no & below 5000 \\
\hline Private Sector & none & no & below 5000 \\
\hline Business & none & no & below 5000 \\
\hline Private Sector & none & no & below 5000 \\
\hline Private Sector & none & no & below 5000 \\
\hline Private Sector & none & no & below 5000 \\
\hline Private Sector & none & no & below 5000 \\
\hline Industrial Sector & none & no & below 5000 \\
\hline Industrial Sector & none & no & below 5000 \\
\hline Private Sector & none & no & below 5000 \\
\hline Industrial Sector & none & no & below 5000 \\
\hline Private Sector & none & no & below 5000 \\
\hline Industrial Sector & none & no & $5000-10000$ \\
\hline Private Sector & none & no & below 5000 \\
\hline Industrial Sector & none & no & below 5000 \\
\hline Business & none & no & $15000-20000$ \\
\hline Foreign & none & no & below 5000 \\
\hline Industrial Sector & none & no & $10000-15000$ \\
\hline Private Sector & none & no & below 5000 \\
\hline Private Sector & none & no & below 5000 \\
\hline Business & none & no & below 5000 \\
\hline Private Sector & none & no & below 5000 \\
\hline Private Sector & none & no & below 5000 \\
\hline Government & none & no & below 5000 \\
\hline Industrial Sector & none & no & below 5000 \\
\hline Industrial Sector & none & no & below 5000 \\
\hline Agriculture & none & no & below 5000 \\
\hline Government & none & no & below 5000 \\
\hline none & none & no & $5000-10000$ \\
\hline Private Sector & none & no & $10000-15000$ \\
\hline Private Sector & none & no & $15000-20000$ \\
\hline Government & none & no & $10000-15000$ \\
\hline Business & none & no & OVER 20000 \\
\hline Industrial Sector & none & yes & $5000-10000$ \\
\hline
\end{tabular}




\begin{tabular}{|c|c|c|c|}
\hline Industrial Sector & none & yes & $5000-10000$ \\
\hline Government & none & no & $15000-20000$ \\
\hline Self Employment & none & yes & $5000-10000$ \\
\hline Self Employment & none & yes & below 5000 \\
\hline Industrial Sector & none & yes & $5000-10000$ \\
\hline Government & none & no & $10000-15000$ \\
\hline Self Employment & none & no & OVER 20000 \\
\hline Private Sector & none & no & OVER 20000 \\
\hline Government & none & no & below 5000 \\
\hline Business & none & no & $15000-20000$ \\
\hline Self Employment & none & yes & $5000-10000$ \\
\hline Business & none & no & OVER 20000 \\
\hline Self Employment & none & no & $15000-20000$ \\
\hline Business & none & no & $15000-20000$ \\
\hline Business & none & no & OVER 20000 \\
\hline Self Employment & none & no & $15000-20000$ \\
\hline Business & none & no & $15000-20000$ \\
\hline Self Employment & none & yes & below 5000 \\
\hline Business & none & no & $10000-15000$ \\
\hline none & none & yes & below 5000 \\
\hline none & none & yes & below 5000 \\
\hline Business & none & no & $10000-15000$ \\
\hline Business & none & no & $5000-10000$ \\
\hline Government & none & no & below 5000 \\
\hline Private Sector & none & yes & $5000-10000$ \\
\hline none & none & no & below 5000 \\
\hline Government & none & no & $5000-10000$ \\
\hline Industrial Sector & none & yes & below 5000 \\
\hline Industrial Sector & none & no & $5000-10000$ \\
\hline Private Sector & none & yes & below 5000 \\
\hline Private Sector & none & no & below 5000 \\
\hline Government & none & no & $5000-10000$ \\
\hline Private Sector & none & no & $5000-10000$ \\
\hline Government & none & no & $5000-10000$ \\
\hline Government & none & no & $5000-10000$ \\
\hline Private Sector & none & no & $5000-10000$ \\
\hline Business & none & no & $5000-10000$ \\
\hline
\end{tabular}




$\begin{array}{cccc}\text { Government } & \text { none } & \text { no } & 5000-10000 \\ \text { Private Sector } & \text { none } & \text { no } & \text { below } 5000 \\ \text { Private Sector } & \text { none } & \text { yes } & \text { below } 5000 \\ \text { Government } & \text { none } & \text { no } & \text { below } 5000 \\ \text { Government } & \text { none } & \text { no } & 5000-10000 \\ \text { Business } & \text { none } & \text { no } & 5000-10000 \\ \text { Private Sector } & \text { none } & \text { no } & 10000-15000\end{array}$


500 intense

2500 intense

7500 intense

7500 intense

2500 intense

2500 intense

2500 intense

2500 intense

7500 intense

17500 intense

7500 intense

2500 intense

2500 intense

12500 intense

2500 intense

7500 intense

2500 intense

2500 intense

12500 intense
Type of the water problem

no continuous water supply

no continuous water supply

no continuous water supply

no continuous water supply

no continuous water supply

no problem

no continuous water supply

no continuous water supply

no continuous water supply

no continuous water supply

no continuous water supply

no continuous water supply

no problem

no continuous water supply

no continuous water supply

no continuous water supply

no continuous water supply

no pure water

no continuous water supply

no continuous water supply

no continuous water supply

no continuous water supply

no problem

no continuous water supply

no continuous water supply

no continuous water supply

no pure water

water problem re

yes

yes

yes

yes

yes

yes

yes

yes

yes

yes

yes

yes

yes

yes

yes

yes

yes

yes

yes

yes

yes

yes

yes

yes

yes

yes

yes

no continuos supply of water and $n$ yes

no continuous water supply yes

no continuous water supply yes

no continuous water supply yes

no continuous water supply yes

no continuous water supply yes

no continuous water supply yes

no continuous water supply yes

no continuous water supply yes

no continuous water supply yes

no continuous water supply yes

no continuous water supply yes

no continuous water supply yes

no continuous water supply yes

no continuous water supply yes

no continuous water supply yes

no problem yes

no continuous water supply yes 


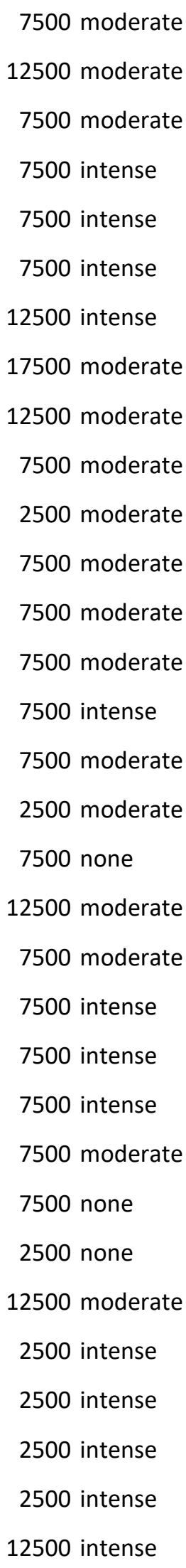

no continuos supply of water and $n$ yes no continuos supply of water and $n$ yes no continuos supply of water and $n$ yes not enough water yes not enough water yes not enough water yes not enough water yes not enough water yes not enough water yes no continuos supply of water and $n$ yes no continuos supply of water and $n$ yes not enough water yes not enough water yes not enough water yes not enough water yes not enough water yes no continuos supply of water and $n$ yes no problem yes not enough water yes not enough water yes not enough water yes not enough water yes no continuos supply of water and $n$ yes not enough water yes no problem yes not enough water yes not enough water yes not enough water yes not enough water yes not enough water yes no continuos supply of water and $n$ yes not enough water yes 


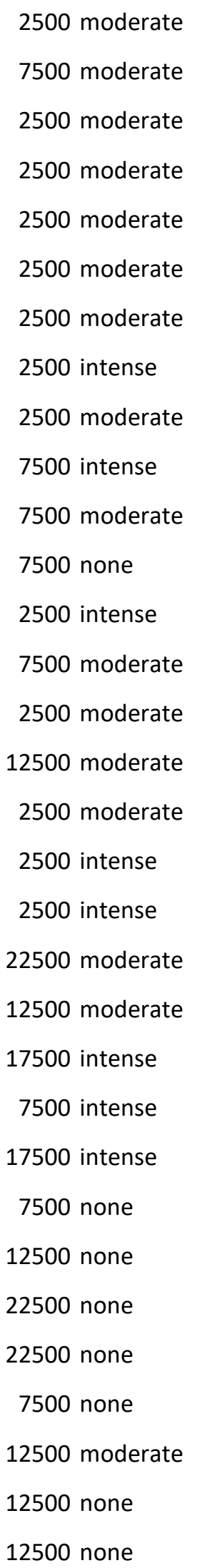

$\begin{array}{lc}\text { not enough water } & \text { yes } \\ \text { not enough water } & \text { yes } \\ \text { not enough water } & \text { yes } \\ \text { not enough water } & \text { yes } \\ \text { not enough water } & \text { yes } \\ \text { not enough water } & \text { yes } \\ \text { not enough water } & \text { yes } \\ \text { not enough water } & \text { yes } \\ \text { not enough water } & \text { yes } \\ \text { not enough water } & \text { yes } \\ \text { not enough water } & \text { yes } \\ \text { no problem } & \text { yes }\end{array}$

no continuos supply of water and $n$ yes

not enough water yes

all yes

all yes

not enough water yes

not enough water yes

no continuous water supply yes

other yes

other yes

no continuous water supply yes

other yes

no continuous water supply yes

no problem no

no problem no

no problem no

no problem yes

no problem no

no pure water yes

no problem no

no problem no 


\begin{tabular}{|c|c|c|}
\hline 22500 none & no problem & no \\
\hline 12500 intense & other & yes \\
\hline 22500 intense & no continuous water supply & yes \\
\hline 17500 intense & other & yes \\
\hline 17500 none & no problem & yes \\
\hline 12500 intense & no pure water & no \\
\hline 22500 moderate & no continuous water supply & yes \\
\hline 12500 none & no problem & no \\
\hline 12500 moderate & no continuous water supply & yes \\
\hline 12500 none & no problem & no \\
\hline 12500 none & no problem & no \\
\hline 22500 moderate & no pure water & no \\
\hline 22500 none & no problem & no \\
\hline 12500 moderate & no continuous water supply & no \\
\hline 22500 none & no problem & yes \\
\hline 7500 intense & not enough water & yes \\
\hline 7500 intense & \multicolumn{2}{|c|}{ no continuos supply of water and $n$ yes } \\
\hline 12500 intense & no continuous water supply & yes \\
\hline 7500 intense & no pure water & yes \\
\hline 7500 intense & all & yes \\
\hline 17500 intense & all & yes \\
\hline 2500 intense & all & yes \\
\hline 2500 intense & no continuous water supply & yes \\
\hline 2500 intense & no continuous water supply & yes \\
\hline 7500 intense & \multicolumn{2}{|c|}{ no continuos supply of water and $n$ yes } \\
\hline 17500 intense & all & yes \\
\hline 7500 intense & all & yes \\
\hline 7500 intense & \multicolumn{2}{|c|}{ no continuos supply of water and $n$ yes } \\
\hline 7500 intense & \multicolumn{2}{|c|}{ no continuos supply of water and $n$ yes } \\
\hline 2500 intense & no pure water & yes \\
\hline 7500 intense & \multicolumn{2}{|c|}{ no continuos supply of water and $n$ yes } \\
\hline 2500 intense & no pure water & yes \\
\hline
\end{tabular}


7500 intense

all

yes

2500 intense

no pure water

yes

2500 intense

no continuous water supply

yes

2500 intense

no continuos supply of water and $n$ yes

2500 intense

all

yes

2500 intense

2500 intense

no continuous water supply yes

2500 intense

no continuos supply of water and $n$ yes

no continuos supply of water and $n$ yes

2500 intense

no continuos supply of water and $n$ yes

2500 intense

no continuos supply of water and $n$ yes

2500 intense

no continuos supply of water and $n$ yes

2500 intense

no continuos supply of water and $n$ yes

2500 intense

2500 intense

2500 intense

7500 intense

2500 intense

2500 intense

17500 intense

2500 intense

12500 intense

2500 intense

2500 intense

2500 intense

2500 intense

2500 intense

2500 intense

2500 intense

2500 intense

2500 intense

2500 intense

7500 none

12500 none

17500 none

12500 none

22500 none

7500 none

no continuous water supply yes

all yes

all yes

all yes

all yes

no continuos supply of water and $n$ yes

no continuos supply of water and $n$ yes

all yes

all yes

all yes

no continuos supply of water and $n$ yes

all yes

no continuos supply of water and $n$ yes

all yes

all yes

all yes

all yes

all yes

all yes

no continuous water supply yes

no continuous water supply yes

no continuous water supply yes

no continuos supply of water and $n$ yes

no problem no

no problem yes 


\begin{tabular}{|c|c|c|}
\hline 7500 none & no problem & no \\
\hline 17500 none & no problem & yes \\
\hline 7500 none & no problem & yes \\
\hline 2500 none & no problem & yes \\
\hline 7500 none & no continuous water supply & yes \\
\hline 12500 none & no continuous water supply & yes \\
\hline 22500 none & no continuous water supply & yes \\
\hline 22500 none & no problem & yes \\
\hline 2500 none & no problem & yes \\
\hline 17500 none & no continuous water supply & yes \\
\hline 7500 none & no problem & yes \\
\hline 22500 none & no problem & yes \\
\hline 17500 none & no continuous water supply & yes \\
\hline 17500 intense & no continuos supply of wate & nyes \\
\hline 22500 moderate & no continuous water supply & yes \\
\hline 17500 none & no problem & yes \\
\hline 17500 none & no problem & yes \\
\hline 2500 none & no continuous water supply & yes \\
\hline 12500 moderate & no continuos supply of wate & nyes \\
\hline 2500 moderate & no continuous water supply & yes \\
\hline 2500 none & no problem & yes \\
\hline 12500 moderate & no continuous water supply & yes \\
\hline 7500 none & no problem & yes \\
\hline 2500 none & no continuous water supply & yes \\
\hline 7500 moderate & no continuous water supply & yes \\
\hline 2500 none & no continuous water supply & yes \\
\hline 7500 none & no continuous water supply & yes \\
\hline 2500 none & no continuous water supply & yes \\
\hline 7500 none & no continuous water supply & yes \\
\hline 2500 none & no problem & yes \\
\hline 2500 moderate & no continuous water supply & yes \\
\hline 7500 moderate & no continuous water supply & yes \\
\hline 7500 none & no problem & yes \\
\hline 7500 none & no problem & yes \\
\hline 7500 none & no problem & yes \\
\hline 7500 moderate & \multicolumn{2}{|c|}{ no continuos supply of water and $n$ yes } \\
\hline 7500 moderate & no continuous water supply & es \\
\hline
\end{tabular}


7500 moderate

2500 intense

2500 moderate

2500 none

7500 none

7500 none

12500 none no continuous water supply

yes

no continuos supply of water and $n$ yes

no continuos supply of water and $n$ yes

no problem

yes

no problem

yes

no problem

yes

no continuous water supply 


\begin{tabular}{|c|c|c|}
\hline do you have a private well? & \multicolumn{2}{|c|}{ is your private drinking water so for domestagriculutre water source } \\
\hline yes & well and tube well & yes \\
\hline yes & well and tube well & yes \\
\hline yes & well and tube well & well \\
\hline yes & well and tube well & yes \\
\hline yes & well and tube well & yes \\
\hline yes & well and tube well & yes \\
\hline yes & well and tube well & well \\
\hline yes & well and tube well & yes \\
\hline yes & well and tube well & yes \\
\hline yes & well and tube well & yes \\
\hline yes & well and tube well & yes \\
\hline yes & well and tube well & yes \\
\hline yes & well and tube well & yes \\
\hline yes & well and tube well & yes \\
\hline yes & well and tube well & yes \\
\hline yes & well and tube well & yes \\
\hline yes & well and tube well & yes \\
\hline yes & well and tube well & yes \\
\hline yes & well and tube well & yes \\
\hline yes & well and tube well & yes \\
\hline yes & well and tube well & yes \\
\hline yes & well and tube well & yes \\
\hline yes & well and tube well & yes \\
\hline yes & well and tube well & yes \\
\hline yes & well and tube well & yes \\
\hline yes & well and tube well & yes \\
\hline yes & well and tube well & yes \\
\hline no & well and tube well & yes \\
\hline yes & well and tube well & yes \\
\hline yes & well and tube well & yes \\
\hline yes & well and tube well & yes \\
\hline yes & well and tube well & yes \\
\hline no & well and tube well & yes \\
\hline yes & well and tube well & yes \\
\hline yes & well and tube well & yes \\
\hline yes & well and tube well & yes \\
\hline yes & well and tube well & yes \\
\hline yes & well and tube well & yes \\
\hline no & well and tube well & yes \\
\hline yes & well and tube well & yes \\
\hline yes & well and tube well & yes \\
\hline yes & well and tube well & yes \\
\hline yes & well and tube well & yes \\
\hline yes & well and tube well & yes \\
\hline yes & well and tube well & well \\
\hline
\end{tabular}




\begin{tabular}{|c|c|c|c|}
\hline yes & well & yes & rain water \\
\hline yes & well & yes & rain water \\
\hline yes & well & yes & rain water \\
\hline yes & well & yes & rain water \\
\hline yes & well & yes & rain water \\
\hline yes & well & yes & \\
\hline yes & well & yes & rain and streams and resı \\
\hline yes & well & yes & rain water \\
\hline yes & well & yes & rain and streams and res, \\
\hline yes & well & yes & rain and streams and resı \\
\hline yes & well & yes & rain and streams and res, \\
\hline yes & well & yes & \\
\hline yes & well & yes & rain and streams and res, \\
\hline yes & well & yes & rain and streams and resı \\
\hline yes & well & yes & rain water \\
\hline yes & well & yes & rain water \\
\hline no & well & yes & rain water \\
\hline yes & well & yes & rain water \\
\hline yes & well & no & \\
\hline no & well & yes & rain and streams and resı \\
\hline yes & well & yes & rain and streams and res, \\
\hline yes & well & yes & rain water \\
\hline yes & well & yes & rain water \\
\hline no & well & yes & rain water \\
\hline yes & well & yes & rain water \\
\hline no & well & yes & \\
\hline no & well & yes & rain water \\
\hline yes & well & yes & rain water \\
\hline yes & well & yes & rain water \\
\hline yes & pipeline & yes & rain water \\
\hline yes & well & yes & rain and streams and resı \\
\hline yes & well & yes & rain water \\
\hline
\end{tabular}




\begin{tabular}{|c|c|c|c|}
\hline no & well & yes & rain water \\
\hline yes & well & yes & rain water \\
\hline no & well & yes & rain water \\
\hline no & tube well & yes & rain and streams and res \\
\hline no & well & yes & rain water \\
\hline no & well & yes & \\
\hline no & tube well & yes & \\
\hline yes & well & yes & \\
\hline no & tube well & yes & rain water \\
\hline yes & well & yes & \\
\hline yes & well & yes & rain water \\
\hline yes & well & yes & \\
\hline no & well & yes & rain water \\
\hline yes & well & yes & rain water \\
\hline yes & well & yes & rain water \\
\hline yes & well & yes & rain water \\
\hline yes & well & yes & rain water \\
\hline yes & well & yes & rain water \\
\hline no & pipeline & yes & \\
\hline yes & pipeline & yes & stream or natural water \\
\hline no & pipeline & yes & \\
\hline yes & pipeline & yes & \\
\hline yes & pipeline & yes & \\
\hline yes & & yes & \\
\hline yes & & yes & \\
\hline yes & & yes & \\
\hline yes & & yes & \\
\hline yes & & yes & \\
\hline yes & & yes & \\
\hline yes & & yes & stream or natural water \\
\hline yes & & yes & \\
\hline yes & & yes & \\
\hline
\end{tabular}




\begin{tabular}{|c|c|c|c|}
\hline yes & & yes & stream or natural water \\
\hline no & & no & \\
\hline no & & no & \\
\hline no & & no & \\
\hline yes & & yes & \\
\hline yes & & no & \\
\hline yes & & no & \\
\hline yes & & yes & \\
\hline yes & & yes & stream or natural water \\
\hline yes & & yes & \\
\hline yes & & yes & \\
\hline yes & & yes & \\
\hline yes & & no & \\
\hline yes & & no & \\
\hline yes & & yes & \\
\hline yes & well & yes & well \\
\hline yes & well & yes & well \\
\hline yes & well & yes & rain water \\
\hline yes & pipeline & yes & rain water \\
\hline no & pipeline & yes & rain water \\
\hline no & stream or natural water & yes & rain water \\
\hline yes & stream or natural water & yes & rain water \\
\hline yes & tube well & yes & rain water \\
\hline yes & well & yes & rain water \\
\hline yes & stream or natural water & yes & well \\
\hline yes & tube well & yes & well \\
\hline yes & tube well & yes & rain water \\
\hline yes & pipeline & yes & rain water \\
\hline yes & pipeline & yes & rain water \\
\hline yes & well & yes & rain water \\
\hline yes & well & yes & rain water \\
\hline yes & well & yes & rain water \\
\hline
\end{tabular}




\begin{tabular}{|c|c|c|c|}
\hline yes & pipeline & yes & stream or natural water \\
\hline no & pipeline & yes & well \\
\hline no & pipeline & yes & \\
\hline no & pipeline & yes & \\
\hline no & well and tube well & yes & \\
\hline no & rain water and pipe line & yes & \\
\hline yes & rain water and pipe line & yes & \\
\hline no & rain water and pipe line & yes & \\
\hline no & pipeline & yes & \\
\hline no & rain water and pipe line & yes & \\
\hline no & pipeline & yes & \\
\hline no & pipeline & yes & \\
\hline no & stream or natural water & yes & \\
\hline yes & wells and rain water & yes & \\
\hline no & pipeline & yes & \\
\hline no & pipeline & yes & \\
\hline no & pipeline & yes & \\
\hline no & pipeline & yes & \\
\hline no & pipeline & yes & \\
\hline no & pipeline & yes & \\
\hline no & pipeline & yes & \\
\hline no & pipeline & yes & \\
\hline no & pipeline & yes & \\
\hline no & stream or natural water & yes & \\
\hline yes & wells and rain water & yes & \\
\hline no & rain water and pipe line & yes & \\
\hline yes & well & yes & \\
\hline yes & wells and rain water & yes & \\
\hline no & stream or natural water & yes & \\
\hline no & all sources & yes & rain and streams and res, \\
\hline no & wells and rain water & yes & \\
\hline yes & tube well & yes & \\
\hline no & pipeline & yes & \\
\hline yes & well & yes & \\
\hline yes & well & yes & \\
\hline yes & well & yes & \\
\hline no & pipeline & yes & \\
\hline
\end{tabular}




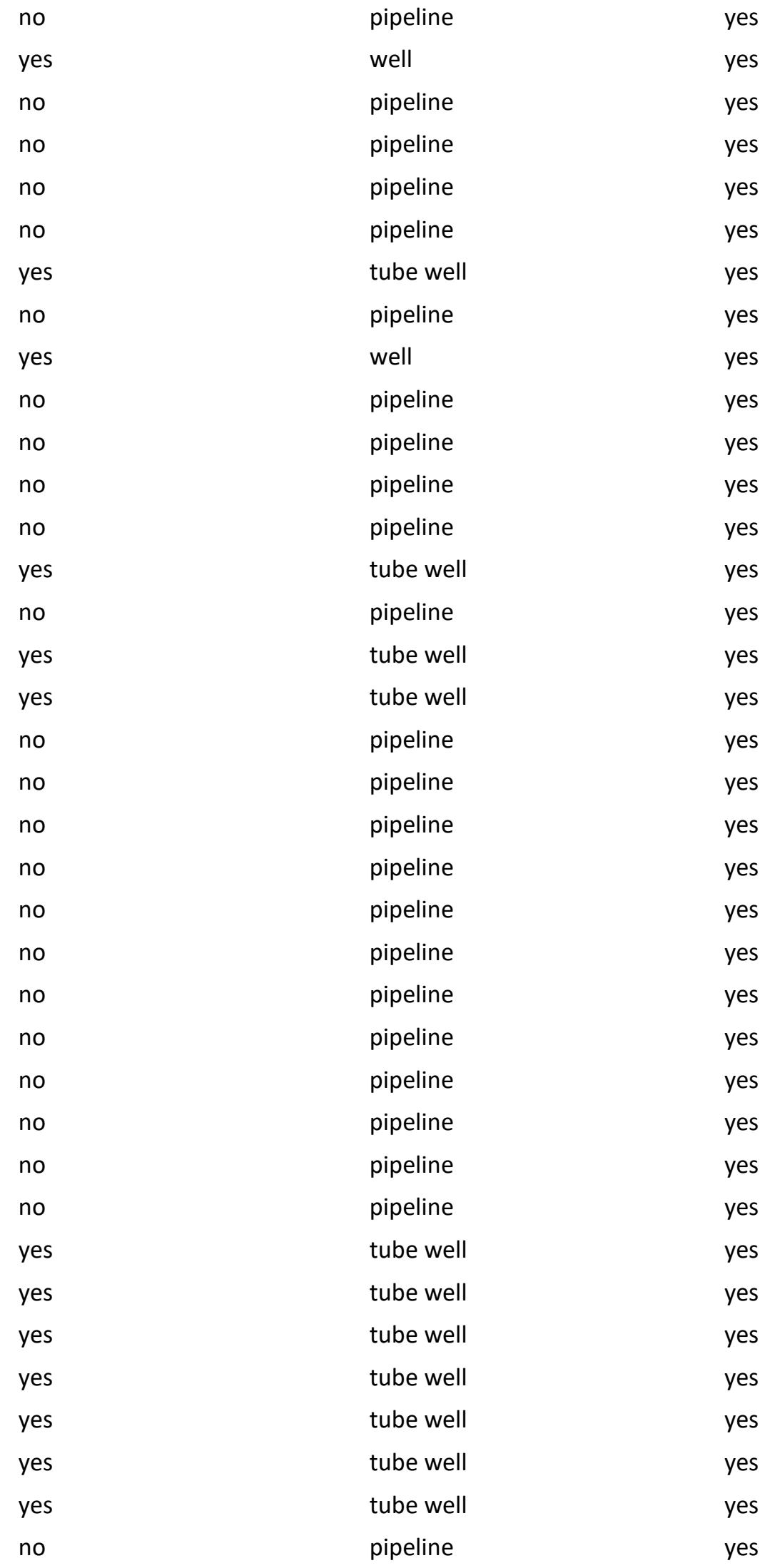


no

no

no

yes

yes

no

no pipeline

pipeline

pipeline

tube well

tube well

pipeline

pipeline yes

yes

yes

yes

yes

yes

yes 
no

no

yes

no

no

no

no

no

no

no

no

no

no

no

no

no

no

no

no

no

no

no

no

no

no

no

no

no

no

no

no

no

no

no

no

no

no

no

no

no

yes well and tube well

well and tube well

well and tube well

well and tube well

well and tube well

well and tube well

well and tube well

well and tube well

well and tube well

well and tube well

well and tube well

well and tube well

well and tube well

well and tube well

well and tube well

well and tube well

well and tube well

well and tube well

well and tube well

well and tube well

well and tube well

well and tube well

well and tube well

well and tube well

well and tube well

well and tube well

well and tube well

well and tube well

well and tube well

well and tube well

well and tube well

well and tube well

well and tube well

well and tube well

well and tube well

well and tube well

well and tube well

well and tube well

well and tube well

well and tube well

well and tube well

well and tube well

well and tube well

well and tube well

well and tube well no

30

no

12

no

no

no

no

yes

no

no

no

no

yes

no

no

no

no

no

no

no

no

no

no

no

no

no

no

no

no

no

no

no

no

no

no

no

no

no

no

no

no

no

no

yes

no

no
16

15

8

10

8

15

30

10

16

12

8

6

6

6

8

12

20

20

12

6

8

18

8

6

9

9

25

21

6

18

12

6

12

15

20

6

6

30

8

15

10

10

15 


\begin{tabular}{|c|c|c|c|}
\hline yes & well & yes & 10 \\
\hline yes & well & yes & 10 \\
\hline yes & well & yes & 10 \\
\hline yes & well & yes & 10 \\
\hline yes & well & yes & 10 \\
\hline no & well & yes & 20 \\
\hline yes & well & yes & 10 \\
\hline yes & well & yes & 10 \\
\hline yes & well & yes & 20 \\
\hline yes & well & yes & 10 \\
\hline yes & well & yes & 10 \\
\hline no & well & yes & 10 \\
\hline yes & stream or natural water & yes & 10 \\
\hline yes & well & yes & 10 \\
\hline yes & well & yes & 10 \\
\hline yes & well & yes & 10 \\
\hline yes & well & yes & 10 \\
\hline yes & well & yes & 10 \\
\hline no & well & yes & 10 \\
\hline yes & well & yes & 20 \\
\hline yes & well & yes & 15 \\
\hline yes & well & yes & 10 \\
\hline yes & well & yes & 15 \\
\hline yes & well & yes & 15 \\
\hline yes & stream or natural water & yes & 15 \\
\hline no & well & yes & 10 \\
\hline yes & well & yes & 10 \\
\hline yes & stream or natural water & yes & 10 \\
\hline yes & well & yes & 10 \\
\hline yes & well & yes & 15 \\
\hline yes & well & yes & 10 \\
\hline yes & well & yes & 10 \\
\hline
\end{tabular}




\begin{tabular}{|c|c|c|c|}
\hline yes & well & yes & 10 \\
\hline yes & well & yes & 10 \\
\hline yes & stream or natural water & yes & 10 \\
\hline yes & stream or natural water & yes & 10 \\
\hline yes & well & yes & 10 \\
\hline no & well & yes & 10 \\
\hline no & well & yes & 10 \\
\hline no & well & yes & 10 \\
\hline yes & well & yes & 10 \\
\hline no & well & yes & 10 \\
\hline yes & well & yes & 15 \\
\hline no & well & yes & 10 \\
\hline yes & well & yes & 10 \\
\hline yes & well & yes & 15 \\
\hline yes & well & yes & 10 \\
\hline yes & well & yes & 10 \\
\hline yes & well & yes & 40 \\
\hline yes & well & yes & 10 \\
\hline no & & no & 10 \\
\hline yes & & no & 10 \\
\hline no & & no & 10 \\
\hline no & & no & 10 \\
\hline no & & no & 10 \\
\hline no & & no & 10 \\
\hline no & & no & 10 \\
\hline no & & no & 10 \\
\hline no & & no & 10 \\
\hline no & & no & 10 \\
\hline no & & no & 15 \\
\hline yes & & no & 10 \\
\hline no & & no & 15 \\
\hline no & & no & 10 \\
\hline
\end{tabular}




\begin{tabular}{|c|c|c|c|}
\hline & no & 10 \\
\hline \multicolumn{2}{|l|}{ no } & no & 10 \\
\hline \multicolumn{2}{|l|}{ no } & no & 20 \\
\hline \multicolumn{2}{|l|}{ no } & no & 10 \\
\hline \multicolumn{2}{|l|}{ no } & no & 10 \\
\hline \multicolumn{2}{|l|}{ no } & no & 20 \\
\hline \multicolumn{2}{|l|}{ no } & no & 10 \\
\hline \multicolumn{2}{|l|}{ no } & no & 10 \\
\hline \multicolumn{2}{|l|}{ yes } & no & 10 \\
\hline \multicolumn{2}{|l|}{ no } & no & 20 \\
\hline \multicolumn{2}{|l|}{ no } & no & 15 \\
\hline \multicolumn{2}{|l|}{ no } & no & 15 \\
\hline \multicolumn{2}{|l|}{ no } & no & 15 \\
\hline \multicolumn{2}{|l|}{ no } & no & 15 \\
\hline \multicolumn{2}{|l|}{ no } & no & 25 \\
\hline yes & well & & 10 \\
\hline yes & well & & 10 \\
\hline yes & pipeline & & 20 \\
\hline yes & rain water & & 10 \\
\hline yes & rain water & & 15 \\
\hline yes & stream or natural water & & 20 \\
\hline yes & pipeline & & 5 \\
\hline yes & stream or natural water & & 20 \\
\hline yes & stream or natural water & & 10 \\
\hline yes & stream or natural water & & 35 \\
\hline yes & stream or natural water & & 5 \\
\hline no & pipeline & & 10 \\
\hline no & pipeline & & 5 \\
\hline no & stream or natural water & & 5 \\
\hline no & well & & 20 \\
\hline no & tube well & & 9 \\
\hline no & well & & 0 \\
\hline
\end{tabular}


$\begin{array}{ll}\text { pipeline } & 10\end{array}$

yes

$\begin{array}{ll}\text { well } & 10\end{array}$

pipeline

stream or natural water

well

stream or natural water 20

well

stream or natural water

well

stream or natural water

stream or natural water 20

stream or natural water

stream or natural water 10

stream or natural water

well

stream or natural water

stream or natural water

stream or natural water

rain water $\quad 20$

$\begin{array}{ll}\text { stream or natural water } & 30\end{array}$

$\begin{array}{ll}\text { stream or natural water } & 20\end{array}$

tube well $\quad 4$

$\begin{array}{ll}\text { pipeline } & 10\end{array}$

$\begin{array}{ll}\text { well } & 10\end{array}$

$\begin{array}{ll}\text { well } & 10\end{array}$

well 10

$\begin{array}{ll}\text { pipeline } & 15\end{array}$ 


\begin{tabular}{|c|c|c|}
\hline no & pipeline & 12 \\
\hline no & well & 5 \\
\hline no & pipeline & 10 \\
\hline no & pipeline & 5 \\
\hline no & pipeline & 8 \\
\hline no & pipeline & 12 \\
\hline no & tube well & 10 \\
\hline no & pipeline & 10 \\
\hline no & well & 4 \\
\hline no & pipeline & 20 \\
\hline no & pipeline & 10 \\
\hline no & tube well & 15 \\
\hline no & pipeline & 10 \\
\hline no & tube well & 8 \\
\hline no & pipeline & 8 \\
\hline no & tube well & 6 \\
\hline no & tube well & 20 \\
\hline no & tube well & 10 \\
\hline no & pipeline & 10 \\
\hline no & pipeline & 2 \\
\hline no & pipeline & 3 \\
\hline no & pipeline & 5 \\
\hline no & pipeline & 12 \\
\hline no & pipeline & 12 \\
\hline no & pipeline & 5 \\
\hline no & pipeline & 5 \\
\hline no & pipeline & 9 \\
\hline no & pipeline & 10 \\
\hline no & pipeline & 15 \\
\hline no & well and tube well & 6 \\
\hline no & well and tube well & 5 \\
\hline no & tube well & 6 \\
\hline no & tube well & 10 \\
\hline no & tube well & 6 \\
\hline no & tube well & 12 \\
\hline no & tube well & 4 \\
\hline no & pipeline & 6 \\
\hline
\end{tabular}




\begin{tabular}{|c|c|c|c|}
\hline no & pipeline & & 6 \\
\hline no & pipeline & & 12 \\
\hline no & pipeline & & 18 \\
\hline no & tube well & & 8 \\
\hline no & well & & 5 \\
\hline no & pipeline & & 6 \\
\hline nc & pipeline & & 10 \\
\hline & & Max & 40 \\
\hline & & Min & 2 \\
\hline & & Mean & 13 \\
\hline & & STDEV & 6.42 \\
\hline & & STDEVP & 6.40 \\
\hline & & Average & 3 \\
\hline
\end{tabular}


f water used for cooking in litely of water for washing pater for toilet usage in I water used or bathing in lit€

25

20

24

9

8

6

12

18

40

12

24

6

6

6

6

10

8

12

18

30

18

8

14

20

8

8

15

18

30

8

24

24

10

6

20

30

40

6

6

20

8

20

6

20

15
50

40

20

20

40

30

12

12

80

20

24

500

12

12

12

16

24

30

16

70

30

16

16

30

250

20

18

12

50

18

40

40

20

20

40

70

60

15

12

40

16

50

15

40

40
30

24

24

40

16

20

12

18

36

30

16

30

20

12

15

8

20

32

20

32

24

24

8

30

40

8

16

16

20

40

8

40

16

30

25

20

35

60

15

32

24

44

25

24

20
300

200

85

400

200

200

210

400

400

80

80

250

500

100

75

125

180

500

240

350

200

200

120

630

800

35

125

150

250

300

80

700

150

100

200

250

300

350

150

300

110

250

250

250

250 


\begin{tabular}{|c|c|c|c|}
\hline 10 & 20 & 20 & 500 \\
\hline 20 & 20 & 30 & 1470 \\
\hline 10 & 10 & 20 & 400 \\
\hline 20 & 10 & 20 & 600 \\
\hline 10 & 20 & 20 & 660 \\
\hline 30 & 25 & 25 & 360 \\
\hline 15 & 40 & 20 & 405 \\
\hline 10 & 10 & 10 & 460 \\
\hline 20 & 50 & 20 & 460 \\
\hline 10 & 20 & 30 & 230 \\
\hline 10 & 20 & 10 & 100 \\
\hline 35 & 15 & 40 & 440 \\
\hline 20 & 20 & 30 & 320 \\
\hline 10 & 20 & 30 & 350 \\
\hline 20 & 30 & 40 & 450 \\
\hline 15 & 25 & 75 & 500 \\
\hline 10 & 10 & 10 & 550 \\
\hline 10 & 10 & 10 & 200 \\
\hline 15 & 25 & 40 & 220 \\
\hline 15 & 20 & 10 & 200 \\
\hline 20 & 25 & 15 & 550 \\
\hline 20 & 30 & 40 & 400 \\
\hline 20 & 35 & 30 & 600 \\
\hline 15 & 20 & 35 & 550 \\
\hline 20 & 30 & 40 & 410 \\
\hline 10 & 20 & 20 & 400 \\
\hline 15 & 25 & 10 & 300 \\
\hline 15 & 20 & 20 & 500 \\
\hline 10 & 30 & 40 & 140 \\
\hline 25 & 10 & 35 & 330 \\
\hline 30 & 10 & 50 & 460 \\
\hline 10 & 10 & 10 & 60 \\
\hline
\end{tabular}




\begin{tabular}{|c|c|c|c|}
\hline 15 & 25 & 20 & 400 \\
\hline 15 & 25 & 25 & 300 \\
\hline 10 & 10 & 10 & 90 \\
\hline 10 & 10 & 10 & 160 \\
\hline 10 & 30 & 10 & 50 \\
\hline 10 & 30 & 10 & 40 \\
\hline 10 & 20 & 10 & 50 \\
\hline 15 & 25 & 20 & 250 \\
\hline 15 & 20 & 25 & 250 \\
\hline 20 & 30 & 30 & 400 \\
\hline 25 & 50 & 20 & 420 \\
\hline 15 & 20 & 45 & 550 \\
\hline 10 & 20 & 20 & 250 \\
\hline 10 & 15 & 25 & 500 \\
\hline 15 & 20 & 15 & 350 \\
\hline 15 & 25 & 10 & 350 \\
\hline 45 & 45 & 40 & 650 \\
\hline 10 & 15 & 20 & 250 \\
\hline 15 & 30 & 30 & 400 \\
\hline 20 & 40 & 40 & 450 \\
\hline 15 & 20 & 30 & 200 \\
\hline 20 & 30 & 30 & 400 \\
\hline 15 & 30 & 20 & 250 \\
\hline 10 & 20 & 20 & 200 \\
\hline 20 & 50 & 30 & 300 \\
\hline 20 & 50 & 30 & 350 \\
\hline 20 & 40 & 30 & 400 \\
\hline 20 & 50 & 30 & 500 \\
\hline 20 & 50 & 30 & 500 \\
\hline 10 & 50 & 20 & 200 \\
\hline 20 & 40 & 40 & 500 \\
\hline 15 & 35 & 30 & 400 \\
\hline
\end{tabular}




\begin{tabular}{|c|c|c|c|}
\hline 15 & 50 & 20 & 250 \\
\hline 15 & 25 & 20 & 200 \\
\hline 25 & 50 & 50 & 600 \\
\hline 10 & 20 & 20 & 250 \\
\hline 10 & 20 & 20 & 200 \\
\hline 25 & 50 & 40 & 750 \\
\hline 20 & 50 & 30 & 350 \\
\hline 10 & 25 & 20 & 300 \\
\hline 10 & 25 & 20 & 350 \\
\hline 50 & 100 & 50 & 550 \\
\hline 25 & 50 & 50 & 750 \\
\hline 25 & 50 & 50 & 700 \\
\hline 20 & 25 & 20 & 250 \\
\hline 20 & 50 & 25 & 350 \\
\hline 50 & 100 & 50 & 850 \\
\hline 20 & 40 & 20 & 300 \\
\hline 15 & 25 & 35 & 60 \\
\hline 25 & 45 & 60 & 80 \\
\hline 20 & 20 & 35 & 200 \\
\hline 10 & 25 & 30 & 100 \\
\hline 15 & 250 & 30 & 70 \\
\hline 25 & 30 & 10 & 150 \\
\hline 25 & 50 & 50 & 200 \\
\hline 20 & 60 & 40 & 200 \\
\hline 40 & 400 & 250 & 150 \\
\hline 10 & 20 & 50 & 60 \\
\hline 15 & 30 & 20 & 200 \\
\hline 270 & 435 & 15 & 70 \\
\hline 270 & 435 & 20 & 35 \\
\hline 30 & 90 & 40 & 550 \\
\hline 50 & 200 & 100 & 500 \\
\hline 90 & 60 & 30 & 110 \\
\hline
\end{tabular}




\begin{tabular}{|c|c|c|c|}
\hline 5 & 60 & 50 & 150 \\
\hline 10 & 40 & 40 & 100 \\
\hline 30 & 30 & 50 & 700 \\
\hline 30 & 20 & 30 & 280 \\
\hline 40 & 30 & 50 & 315 \\
\hline 20 & 30 & 50 & 600 \\
\hline 30 & 40 & 60 & 735 \\
\hline 20 & 30 & 40 & 630 \\
\hline 50 & 40 & 40 & 720 \\
\hline 10 & 30 & 15 & 300 \\
\hline 10 & 30 & 20 & 600 \\
\hline 20 & 40 & 20 & 1250 \\
\hline 20 & 20 & 40 & 890 \\
\hline 30 & 80 & 70 & 700 \\
\hline 10 & 45 & 20 & 240 \\
\hline 20 & 25 & 35 & 825 \\
\hline 25 & 20 & 35 & 640 \\
\hline 30 & 50 & 30 & 480 \\
\hline 15 & 35 & 50 & 150 \\
\hline 25 & 40 & 60 & 800 \\
\hline 20 & 50 & 80 & 1050 \\
\hline 10 & 20 & 30 & 420 \\
\hline 20 & 30 & 40 & 720 \\
\hline 30 & 40 & 40 & 525 \\
\hline 20 & 20 & 30 & 420 \\
\hline 30 & 20 & 50 & 840 \\
\hline 20 & 10 & 20 & 420 \\
\hline 20 & 10 & 20 & 420 \\
\hline 20 & 20 & 30 & 420 \\
\hline 40 & 40 & 60 & 525 \\
\hline 20 & 30 & 40 & 600 \\
\hline 10 & 20 & 8 & 150 \\
\hline 10 & 60 & 30 & 250 \\
\hline 5 & 60 & 30 & 150 \\
\hline 20 & 30 & 10 & 200 \\
\hline 20 & 60 & 20 & 400 \\
\hline 30 & 70 & 40 & 600 \\
\hline
\end{tabular}




\begin{tabular}{|c|c|c|c|}
\hline 20 & 50 & 40 & 500 \\
\hline 10 & 30 & 10 & 200 \\
\hline 20 & 50 & 30 & 400 \\
\hline 10 & 20 & 10 & 150 \\
\hline 10 & 20 & 10 & 200 \\
\hline 20 & 50 & 40 & 500 \\
\hline 20 & 30 & 30 & 250 \\
\hline 20 & 40 & 20 & 400 \\
\hline 10 & 20 & 8 & 200 \\
\hline 20 & 60 & 40 & 500 \\
\hline 20 & 50 & 30 & 300 \\
\hline 80 & 60 & 50 & 500 \\
\hline 20 & 40 & 30 & 400 \\
\hline 3 & 50 & 35 & 400 \\
\hline 15 & 30 & 25 & 250 \\
\hline 6 & 300 & 75 & 500 \\
\hline 30 & 50 & 50 & 500 \\
\hline 15 & 25 & 30 & 300 \\
\hline 20 & 40 & 35 & 400 \\
\hline 3 & 8 & 10 & 30 \\
\hline 3 & 6 & 8 & 30 \\
\hline 7 & 20 & 12 & 180 \\
\hline 8 & 30 & 40 & 250 \\
\hline 20 & 30 & 20 & 250 \\
\hline 5 & 30 & 12 & 80 \\
\hline 3 & 20 & 10 & 50 \\
\hline 3 & 30 & 30 & 300 \\
\hline 10 & 35 & 30 & 200 \\
\hline 10 & 75 & 40 & 700 \\
\hline 6 & 20 & 20 & 200 \\
\hline 7 & 18 & 10 & 200 \\
\hline 10 & 30 & 15 & 200 \\
\hline 12 & 30 & 25 & 300 \\
\hline 12 & 20 & 25 & 300 \\
\hline 25 & 40 & 30 & 300 \\
\hline 10 & 20 & 24 & 200 \\
\hline 12 & 18 & 15 & 250 \\
\hline
\end{tabular}




$\begin{array}{cccc}6 & 18 & 32 & 250 \\ 12 & 24 & 30 & 400 \\ 6 & 24 & 40 & 500 \\ 4 & 30 & 30 & 500 \\ 5 & 30 & 30 & 300 \\ 6 & 12 & 6 & 300 \\ 20 & 50 & 30 & 400 \\ & & & \\ 270 & 500 & 250 & 1470 \\ 3 & 6 & 6 & 30 \\ 20 & 43 & 30 & 350 \\ 26.44 & 64.15 & 20.98 & 221.76 \\ 26.38 & 64.00 & 20.94 & 221.26\end{array}$




\section{Total water}

435

296

169

484

272

266

254

463

586

152

160

798

546

136

114

165

240

586

314

502

284

254

166

728

1106

77

183

205

375

387

158

822

208

162

297

385

455

437

189

422

166

379

306

344

340 itlu used source for daily choter quanitity used by boiling i to purify wharcoal to $p$

well and tube well

well and tube well

well and tube well

well and tube well

well and tube well

well and tube well

well and tube well

well and tube well

well and tube well

well and tube well

well and tube well

well and tube well

well and tube well

well and tube well

well and tube well

well and tube well

well and tube well

well and tube well

well and tube well

well and tube well

well and tube well

well and tube well

well and tube well

well and tube well

well and tube well

well and tube well

well and tube well

well and tube well

well and tube well

well and tube well

well and tube well

well and tube well

well and tube well

well and tube well

well and tube well

well and tube well

well and tube well

well and tube well

well and tube well

well and tube well

well and tube well

well and tube well

well and tube well

well and tube well

well and tube well

\begin{tabular}{|c|c|c|c|}
\hline & yes & yes & no \\
\hline & no & no & no \\
\hline & yes & yes & no \\
\hline & yes & yes & no \\
\hline & no & yes & no \\
\hline & yes & no & no \\
\hline & no & no & no \\
\hline & no & no & no \\
\hline & yes & no & no \\
\hline & no & yes & no \\
\hline & yes & no & no \\
\hline & yes & no & no \\
\hline & no & no & no \\
\hline & no & no & no \\
\hline & no & no & no \\
\hline & yes & yes & no \\
\hline & yes & yes & no \\
\hline & no & no & no \\
\hline & no & no & no \\
\hline 402 & yes & no & no \\
\hline 284 & yes & no & no \\
\hline 254 & no & yes & no \\
\hline 166 & yes & no & no \\
\hline 728 & yes & yes & no \\
\hline 876 & yes & no & no \\
\hline 77 & yes & yes & no \\
\hline & yes & no & no \\
\hline & yes & no & no \\
\hline & yes & no & no \\
\hline 387 & yes & no & no \\
\hline 238 & yes & no & no \\
\hline & yes & no & no \\
\hline 208 & yes & no & no \\
\hline & no & no & no \\
\hline & no & no & no \\
\hline & yes & no & no \\
\hline & no & no & no \\
\hline 437 & yes & no & no \\
\hline 189 & no & no & no \\
\hline 422 & no & no & yes \\
\hline 166 & no & no & no \\
\hline 270 & yes & yes & no \\
\hline & no & no & no \\
\hline 344 & yes & no & no \\
\hline 340 & & & \\
\hline
\end{tabular}




\begin{tabular}{|c|c|c|c|c|c|}
\hline 560 & well & 560 & yes & no & no \\
\hline 1550 & well & 1550 & yes & no & no \\
\hline 450 & well & & yes & no & no \\
\hline 660 & well & & yes & no & no \\
\hline 720 & well & & yes & no & no \\
\hline 460 & well & & yes & no & no \\
\hline 490 & well & & yes & no & no \\
\hline 500 & well & & yes & yes & no \\
\hline 570 & well & & yes & yes & no \\
\hline 300 & well & & yes & yes & no \\
\hline 150 & well & & yes & yes & no \\
\hline 540 & well & & yes & yes & no \\
\hline 400 & well & & yes & yes & no \\
\hline 420 & well & & yes & yes & no \\
\hline 550 & well & & yes & yes & no \\
\hline 625 & well & & yes & yes & no \\
\hline 590 & well & & yes & yes & no \\
\hline 240 & well & & yes & yes & no \\
\hline 310 & well & & yes & yes & no \\
\hline 265 & well & & no & no & no \\
\hline 625 & well & & yes & yes & no \\
\hline 500 & well & & yes & yes & no \\
\hline 700 & well & & yes & no & no \\
\hline 635 & well & & no & no & no \\
\hline 515 & stream or natural water & & no & no & no \\
\hline 460 & well & & yes & yes & no \\
\hline 360 & well & & yes & yes & no \\
\hline 565 & stream or natural water & & yes & yes & no \\
\hline 230 & well & & yes & yes & no \\
\hline 415 & well & & yes & yes & no \\
\hline 560 & well & & yes & yes & no \\
\hline 100 & well & & yes & yes & no \\
\hline
\end{tabular}




\begin{tabular}{|c|c|}
\hline 470 & well \\
\hline 375 & well \\
\hline 130 & stream or natural water \\
\hline 200 & stream or natural water \\
\hline 110 & well \\
\hline 100 & well \\
\hline 100 & well \\
\hline 320 & well \\
\hline 320 & well \\
\hline 490 & well \\
\hline 530 & well \\
\hline 640 & well \\
\hline 310 & well \\
\hline 565 & well \\
\hline 410 & well \\
\hline 410 & well \\
\hline 820 & well \\
\hline 305 & well \\
\hline 485 & \\
\hline 560 & \\
\hline 275 & \\
\hline 490 & \\
\hline 325 & \\
\hline 260 & \\
\hline 410 & \\
\hline 460 & \\
\hline 500 & \\
\hline 610 & \\
\hline 615 & \\
\hline 290 & \\
\hline 615 & \\
\hline 490 & \\
\hline
\end{tabular}

\begin{tabular}{|c|c|}
\hline yes & yes \\
\hline yes & yes \\
\hline yes & yes \\
\hline yes & yes \\
\hline yes & yes \\
\hline yes & yes \\
\hline no & no \\
\hline no & no \\
\hline yes & yes \\
\hline yes & yes \\
\hline yes & yes \\
\hline yes & no \\
\hline yes & no \\
\hline yes & no \\
\hline yes & yes \\
\hline no & no \\
\hline yes & yes \\
\hline yes & yes \\
\hline yes & no \\
\hline no & yes \\
\hline yes & no \\
\hline yes & yes \\
\hline yes & yes \\
\hline yes & no \\
\hline yes & no \\
\hline no & no \\
\hline no & yes \\
\hline yes & no \\
\hline yes & no \\
\hline yes & no \\
\hline yes & yes \\
\hline yes & no \\
\hline
\end{tabular}




yes yes no

yes yes no

yes no no

yes yes no

yes no no

yes yes no

yes yes no

yes yes no

yes no no

yes yes no

yes no no

yes no no

yes yes no

yes no no

yes no no

yes no no

yes yes no

yes no no

yes no no

yes yes no

yes yes no

yes yes no

yes yes no

yes yes no

yes yes no

yes yes no

yes yes no

yes yes no

yes yes no

yes yes no

yes no no

yes no no

yes no no

yes yes no

yes no no

no no no




\begin{tabular}{|c|c|c|c|}
\hline 622 & pipeline & no & no \\
\hline 255 & well & yes & no \\
\hline 510 & pipeline & no & no \\
\hline 195 & pipeline & no & no \\
\hline 248 & pipeline & no & no \\
\hline 622 & pipeline & no & no \\
\hline 340 & tube well & no & no \\
\hline 490 & pipeline & yes & no \\
\hline 242 & well & no & no \\
\hline 640 & pipeline & yes & no \\
\hline 410 & pipeline & yes & no \\
\hline 705 & tube well & yes & no \\
\hline 500 & pipeline & yes & no \\
\hline 496 & tube well & yes & yes \\
\hline 328 & pipeline & no & no \\
\hline 887 & tube well & no & no \\
\hline 650 & tube well & yes & yes \\
\hline 380 & tube well & no & no \\
\hline 505 & pipeline & no & no \\
\hline 53 & pipeline & no & no \\
\hline 50 & pipeline & yes & no \\
\hline 224 & pipeline & yes & no \\
\hline 340 & pipeline & yes & no \\
\hline 332 & pipeline & yes & no \\
\hline 132 & pipeline & yes & no \\
\hline 88 & pipeline & yes & no \\
\hline 372 & pipeline & no & no \\
\hline 285 & pipeline & yes & no \\
\hline 840 & pipeline & no & no \\
\hline 252 & well and tube well & yes & no \\
\hline 240 & well and tube well & no & no \\
\hline 261 & tube well & no & no \\
\hline 377 & tube well & yes & no \\
\hline 363 & tube well & yes & no \\
\hline 407 & tube well & no & no \\
\hline 258 & tube well & no & no \\
\hline 301 & pipeline & no & no \\
\hline
\end{tabular}




$\begin{array}{lcccc}312 & \text { pipeline } & \text { no } & \text { no } & \text { no } \\ 478 & \text { pipeline } & \text { yes } & \text { no } & \text { no } \\ 588 & \text { pipeline } & \text { yes } & \text { no } & \text { no } \\ 572 & \text { tube well } & \text { yes } & \text { no } & \text { no } \\ 370 & \text { well } & \text { yes } & \text { yes } & \text { no } \\ 330 & \text { pipeline } & \text { yes } & \text { no } & \text { no } \\ 510 & \text { pipeline } & \text { yes } & \text { no } & \text { no }\end{array}$

1550

50

456

248.67

248.11

110

375.6550218

12.11790393 
ding kekeurrification is ays of purifaste water vater to a n:er to a natı a methodicways of disposal?

\begin{tabular}{|c|c|c|c|c|c|c|c|c|}
\hline no & no & no & yes & yes & no & yes & no & Water sealed \\
\hline no & yes & no & yes & no & no & no & no & Water sealed \\
\hline no & no & no & yes & no & no & no & no & Water sealed \\
\hline no & no & no & yes & no & no & no & no & Water sealed \\
\hline no & no & no & yes & yes & no & no & no & Water sealed \\
\hline no & no & no & yes & yes & no & no & no & Water sealed \\
\hline no & yes & no & yes & yes & no & no & no & Water sealed \\
\hline no & yes & no & yes & no & no & no & no & Water sealed \\
\hline no & no & no & yes & no & no & no & no & Water sealed \\
\hline no & no & no & yes & yes & no & no & no & Water sealed \\
\hline no & no & no & yes & yes & no & no & no & Water sealed \\
\hline no & no & no & yes & no & no & no & no & Water sealed \\
\hline no & yes & no & yes & no & no & no & no & Water sealed \\
\hline no & yes & no & yes & no & no & no & no & neighbor's \\
\hline no & yes & no & yes & no & no & no & no & none \\
\hline no & no & no & yes & yes & no & no & no & Water sealed \\
\hline no & no & no & yes & no & no & no & no & Water sealed \\
\hline no & yes & no & yes & yes & no & no & no & Water sealed \\
\hline no & yes & no & yes & yes & no & no & no & Water sealed \\
\hline no & no & no & yes & no & no & no & no & Water sealed \\
\hline no & no & no & yes & yes & no & no & no & Water sealed \\
\hline no & no & no & yes & no & no & no & no & Water sealed \\
\hline no & no & no & yes & yes & no & no & no & Water sealed \\
\hline no & no & no & yes & yes & no & no & no & Water sealed \\
\hline no & no & no & yes & no & no & no & no & Water sealed \\
\hline no & no & no & yes & no & no & no & no & Water sealed \\
\hline no & no & no & yes & yes & no & no & no & Water sealed \\
\hline no & no & no & no & no & no & no & no & neighbor's \\
\hline no & no & no & no & no & no & no & no & Water sealed \\
\hline no & no & no & yes & yes & no & no & no & Water sealed \\
\hline no & no & no & yes & no & no & no & no & neighbor's \\
\hline no & no & no & yes & no & no & no & no & Water sealed \\
\hline no & no & no & yes & no & no & no & no & neighbor's \\
\hline no & yes & no & yes & no & no & no & no & Water sealed \\
\hline no & yes & no & yes & no & no & no & no & Water sealed \\
\hline no & no & no & yes & no & no & no & no & Water sealed \\
\hline no & yes & no & yes & no & no & no & no & Water sealed \\
\hline no & no & no & yes & no & no & no & no & Water sealed \\
\hline no & yes & no & yes & no & no & no & no & Water sealed \\
\hline no & no & no & yes & no & no & no & no & Water sealed \\
\hline no & yes & no & yes & no & no & no & no & Water sealed \\
\hline no & no & no & no & no & no & yes & no & Water sealed \\
\hline no & yes & no & yes & no & no & no & no & Water sealed \\
\hline no & no & no & yes & yes & no & no & no & Water sealed \\
\hline no & yes & no & yes & yes & no & no & no & Water sealed \\
\hline
\end{tabular}




\begin{tabular}{|c|c|c|c|c|c|c|c|c|}
\hline no & no & no & yes & no & no & no & no & Water sealed \\
\hline no & no & no & yes & no & no & no & no & Water sealed \\
\hline no & no & no & yes & no & no & no & no & Water sealed \\
\hline no & no & no & yes & no & no & no & no & Water sealed \\
\hline no & no & no & yes & no & no & no & no & Water sealed \\
\hline no & no & no & yes & no & no & no & no & Water sealed \\
\hline no & no & no & yes & no & no & no & no & Water sealed \\
\hline no & no & no & yes & no & no & no & no & Water sealed \\
\hline no & no & no & yes & no & no & no & no & Water sealed \\
\hline no & no & no & yes & yes & no & no & no & Water sealed \\
\hline no & no & no & yes & yes & no & no & no & Water sealed \\
\hline no & no & no & yes & yes & no & no & no & Water sealed \\
\hline no & no & no & yes & yes & no & no & no & Water sealed \\
\hline no & no & no & yes & no & no & no & no & Water sealed \\
\hline no & no & no & yes & no & no & no & no & Water sealed \\
\hline no & no & no & yes & no & no & no & no & Water sealed \\
\hline no & no & no & yes & no & no & no & no & Water sealed \\
\hline no & no & no & yes & no & no & no & no & Water sealed \\
\hline no & no & no & yes & yes & no & no & no & Water sealed \\
\hline no & yes & no & yes & yes & no & no & no & Water sealed \\
\hline no & no & no & yes & yes & no & no & no & Water sealed \\
\hline no & no & no & yes & yes & no & no & no & Water sealed \\
\hline no & no & no & yes & yes & no & no & no & Water sealed \\
\hline no & no & no & yes & yes & no & no & no & Water sealed \\
\hline no & no & no & yes & yes & no & no & no & Water sealed \\
\hline no & no & no & yes & no & no & no & no & Water sealed \\
\hline no & no & no & yes & no & no & no & no & Water sealed \\
\hline no & no & no & yes & no & no & no & no & Water sealed \\
\hline no & no & no & yes & no & no & no & no & Water sealed \\
\hline no & no & no & yes & no & no & no & no & Water sealed \\
\hline no & no & no & yes & no & no & no & no & Water sealed \\
\hline no & no & no & yes & no & no & no & no & Water sealed \\
\hline
\end{tabular}




\begin{tabular}{|c|c|c|c|c|c|c|c|c|}
\hline no & no & no & yes & no & no & no & no & Water sealed \\
\hline no & no & no & yes & no & no & no & no & Water sealed \\
\hline no & no & no & yes & no & no & no & no & Water sealed \\
\hline no & no & no & yes & yes & no & no & no & Water sealed \\
\hline no & no & no & yes & yes & no & no & no & Water sealed \\
\hline no & no & no & yes & no & no & no & no & Water sealed \\
\hline no & yes & no & yes & no & no & no & no & Water sealed \\
\hline no & yes & no & yes & yes & no & no & no & Water sealed \\
\hline no & no & no & yes & yes & no & no & no & Water sealed \\
\hline no & no & no & yes & yes & no & no & no & Water sealed \\
\hline no & no & no & yes & yes & no & no & no & Water sealed \\
\hline no & no & no & yes & yes & yes & no & no & Water sealed \\
\hline no & no & no & yes & no & yes & no & no & Water sealed \\
\hline no & no & no & yes & no & yes & no & no & Water sealed \\
\hline no & no & no & yes & no & yes & no & no & Water sealed \\
\hline no & no & no & yes & no & no & no & no & Water sealed \\
\hline no & no & no & yes & no & no & no & no & Water sealed \\
\hline no & no & no & yes & no & no & no & no & Water sealed \\
\hline no & no & no & yes & no & yes & no & no & Water sealed \\
\hline no & no & no & yes & no & no & yes & no & Water sealed \\
\hline no & no & no & yes & yes & no & no & no & Water sealed \\
\hline no & no & no & yes & no & no & yes & no & Water sealed \\
\hline no & no & no & yes & no & yes & no & no & Water sealed \\
\hline no & no & no & yes & no & no & yes & no & Water sealed \\
\hline no & no & no & yes & no & no & yes & no & Water sealed \\
\hline no & yes & no & yes & no & no & yes & no & Water sealed \\
\hline no & no & no & yes & no & no & yes & no & Water sealed \\
\hline no & no & no & yes & no & no & yes & no & Water sealed \\
\hline no & no & no & yes & no & no & yes & no & Water sealed \\
\hline no & no & no & yes & yes & no & no & no & Water sealed \\
\hline no & no & no & yes & yes & no & no & no & Water sealed \\
\hline no & no & no & yes & yes & no & no & no & Water sealed \\
\hline
\end{tabular}




\begin{tabular}{|c|c|c|c|c|c|c|c|c|}
\hline no & no & no & yes & yes & no & no & no & Water sealed \\
\hline no & no & no & yes & yes & no & no & no & Water sealed \\
\hline no & no & no & yes & yes & no & no & no & Water sealed \\
\hline no & no & no & yes & yes & no & no & no & Water sealed \\
\hline no & no & no & yes & yes & no & no & no & Water sealed \\
\hline no & no & no & yes & yes & no & no & no & Water sealed \\
\hline no & no & no & yes & no & no & yes & no & Water sealed \\
\hline no & no & no & yes & yes & no & no & no & Water sealed \\
\hline no & no & no & yes & yes & no & no & no & Water sealed \\
\hline no & no & no & yes & yes & no & no & no & Water sealed \\
\hline no & no & no & yes & yes & no & no & no & Water sealed \\
\hline no & no & no & yes & no & no & yes & no & Water sealed \\
\hline no & no & no & yes & no & no & yes & no & Water sealed \\
\hline no & no & no & yes & no & no & yes & no & Water sealed \\
\hline no & no & no & yes & no & no & yes & no & Water sealed \\
\hline no & no & no & no & yes & no & no & no & Water sealed \\
\hline no & no & no & yes & no & no & no & no & Water sealed \\
\hline no & no & no & yes & no & yes & no & no & Water sealed \\
\hline no & no & no & yes & no & no & no & no & Water sealed \\
\hline no & no & no & yes & no & no & no & no & Water sealed \\
\hline no & no & no & yes & no & yes & no & no & pit \\
\hline no & no & no & no & no & yes & no & no & Water sealed \\
\hline no & yes & no & yes & no & no & yes & no & Water sealed \\
\hline no & & no & yes & yes & no & no & no & Water sealed \\
\hline no & yes & no & yes & yes & no & no & no & Water sealed \\
\hline no & no & no & yes & no & yes & no & no & Water sealed \\
\hline no & no & no & yes & no & yes & no & no & Water sealed \\
\hline no & no & no & yes & no & no & yes & no & Water sealed \\
\hline no & no & no & yes & no & no & yes & no & Water sealed \\
\hline no & no & no & yes & no & no & yes & no & Water sealed \\
\hline no & no & no & yes & no & no & yes & no & Water sealed \\
\hline no & no & no & yes & no & yes & no & no & Water sealed \\
\hline
\end{tabular}




\begin{tabular}{|c|c|c|c|c|c|c|c|c|}
\hline no & no & no & yes & no & no & yes & no & Water sealed \\
\hline no & no & no & yes & no & no & no & no & Water sealed \\
\hline no & no & no & yes & no & no & no & no & Water sealed \\
\hline no & no & no & yes & no & no & no & no & Water sealed \\
\hline no & no & no & yes & no & no & no & no & Water sealed \\
\hline no & no & no & yes & no & no & no & no & Water sealed \\
\hline no & no & no & yes & no & no & no & no & Water sealed \\
\hline no & no & no & yes & no & no & no & no & Water sealed \\
\hline no & no & no & yes & no & no & no & no & Water sealed \\
\hline no & no & no & yes & no & no & yes & no & Water sealed \\
\hline no & no & no & yes & no & no & no & no & Water sealed \\
\hline no & no & no & yes & no & no & no & no & Water sealed \\
\hline no & no & no & yes & no & no & no & no & Water sealed \\
\hline no & no & no & yes & no & no & no & no & Water sealed \\
\hline no & no & no & yes & no & no & no & no & Water sealed \\
\hline no & no & no & yes & no & no & no & no & Water sealed \\
\hline no & no & no & yes & no & no & yes & no & Water sealed \\
\hline no & no & no & yes & no & no & no & no & Water sealed \\
\hline no & no & no & yes & no & no & no & no & Water sealed \\
\hline no & no & no & yes & no & no & no & no & Water sealed \\
\hline no & no & no & yes & no & no & no & no & Water sealed \\
\hline no & no & no & yes & no & no & no & no & Water sealed \\
\hline no & no & no & yes & no & no & no & no & Water sealed \\
\hline no & no & no & yes & no & no & no & no & Water sealed \\
\hline no & no & no & yes & no & no & no & no & Water sealed \\
\hline no & no & no & yes & no & no & no & no & Water sealed \\
\hline no & no & no & yes & no & no & no & no & Water sealed \\
\hline no & no & no & yes & no & no & no & no & Water sealed \\
\hline no & no & no & yes & no & no & no & no & Water sealed \\
\hline no & no & no & yes & no & no & no & no & Water sealed \\
\hline no & no & no & yes & no & no & no & no & Water sealed \\
\hline no & no & no & no & yes & no & no & no & Water sealed \\
\hline no & no & no & no & yes & no & no & no & Water sealed \\
\hline no & no & no & yes & yes & no & no & no & neighbor's \\
\hline no & no & no & no & yes & no & no & no & Water sealed \\
\hline no & no & no & no & yes & no & no & no & Water sealed \\
\hline no & yes & no & no & yes & no & no & no & Water sealed \\
\hline
\end{tabular}




\begin{tabular}{|c|c|c|c|c|c|c|c|c|}
\hline no & no & no & no & yes & no & no & no & Water sealed \\
\hline no & no & no & no & yes & no & no & no & Water sealed \\
\hline no & no & no & no & yes & no & no & no & Water sealed \\
\hline no & no & no & no & yes & no & no & no & Water sealed \\
\hline no & no & no & no & yes & no & no & no & Water sealed \\
\hline no & no & no & no & yes & no & no & no & Water sealed \\
\hline no & no & no & no & yes & no & no & no & Water sealed \\
\hline no & no & no & no & yes & no & no & no & Water sealed \\
\hline no & no & no & no & yes & no & no & no & Water sealed \\
\hline no & no & no & no & yes & no & no & no & Water sealed \\
\hline no & no & no & no & yes & no & no & no & Water sealed \\
\hline no & no & no & no & yes & no & no & no & Water sealed \\
\hline no & no & no & no & yes & no & no & no & Water sealed \\
\hline no & no & no & no & yes & no & no & no & Water sealed \\
\hline no & yes & no & no & yes & no & no & no & Water sealed \\
\hline no & yes & no & no & yes & no & no & no & Water sealed \\
\hline no & no & no & no & yes & no & no & no & Water sealed \\
\hline no & yes & no & no & yes & no & no & no & Water sealed \\
\hline no & yes & no & no & yes & no & no & no & Water sealed \\
\hline no & yes & no & no & yes & no & no & no & Water sealed \\
\hline no & no & no & no & yes & no & no & no & Water sealed \\
\hline no & no & no & no & yes & no & no & no & Water sealed \\
\hline no & no & no & no & yes & no & no & no & Water sealed \\
\hline no & no & no & no & yes & no & no & no & Water sealed \\
\hline no & no & no & no & yes & no & no & no & Water sealed \\
\hline no & no & no & no & yes & no & no & no & Water sealed \\
\hline no & yes & no & no & yes & no & no & no & Water sealed \\
\hline no & no & no & no & yes & no & no & no & Water sealed \\
\hline no & yes & no & no & yes & no & no & no & Water sealed \\
\hline no & no & no & no & yes & no & no & no & Water sealed \\
\hline no & yes & no & no & no & no & yes & no & Water sealed \\
\hline no & yes & no & no & no & no & yes & no & Water sealed \\
\hline no & no & no & no & no & no & yes & no & Water sealed \\
\hline no & no & no & no & no & no & yes & no & Water sealed \\
\hline no & yes & no & no & no & no & yes & no & Water sealed \\
\hline no & yes & no & no & no & yes & no & no & Water sealed \\
\hline no & yes & no & no & yes & no & no & no & Water sealed \\
\hline
\end{tabular}




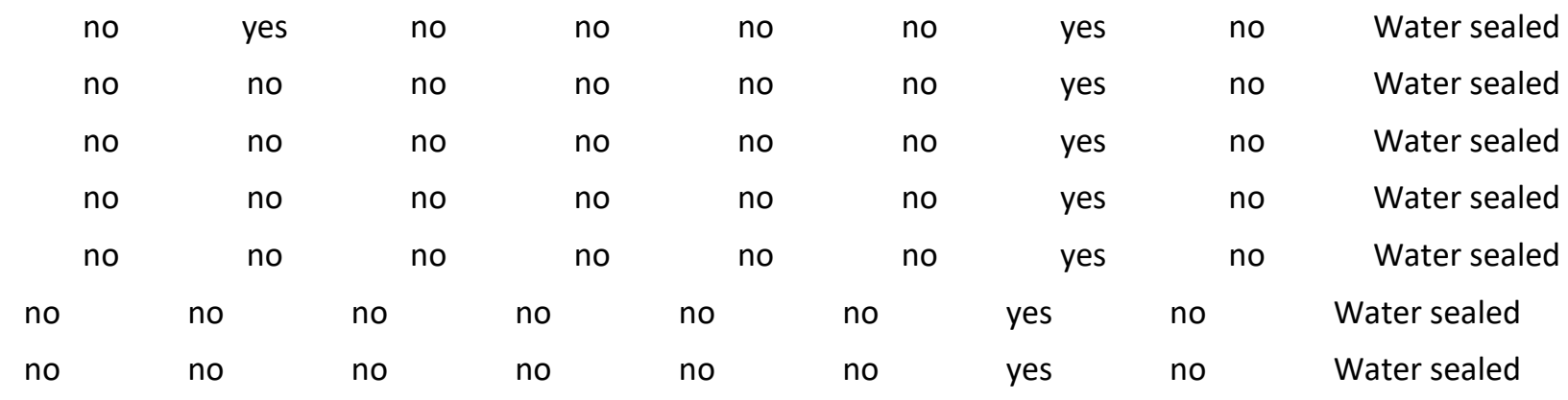

